\title{
O Uso de Simuladores no Ensino da Medicina
}

\author{
The Use of Simulation in Medical Education
}

\author{
Cecilia Dias Flores \\ PPG Ciências da Saúde- UFCSPA \\ Rua Sarmento Leite, 245 \\ Porto Alegre, RS - Brasil \\ dflores@ufcspa.edu.br
}

\author{
Marta Rosecler Bez \\ Universidade Feevale \\ Inst. Ciências Exatas e Tecnológicas \\ Novo Hamburgo, RS - Brasil \\ martabez@,feevale.br
}

\author{
Rosana Mussoi Bruno \\ Dpto. Clínica Médica - UFCSPA \\ Rua Sarmento Leite, 245 \\ Porto Alegre, RS - Brasil \\ rosanab@ufcspa.edu.br
}

\begin{abstract}
Resumo Este trabalho descreve o desenvolvimento de simulação de casos clínicos em um ambiente computadorizado na web. O simulador foi inserido no Moodle e 18 alunos do 7 o semestre de medicina executaram um caso e responderam a um questionário validado para a avaliação de softwares educativos com perguntas sobre: o aprendizado, a interatividade, motivação, concentração, atratividade, operacionalidade e autonomia. A experiência foi considerada extremamente válida. O desafio é enorme no sentido de buscar alternativas de interatividade que estimulem e que simultaneamente abriguem conteúdo, com um aprofundamento adequado ao objetivo do curso. As críticas na avaliação servirão de base para ajustes nos próximos casos.
\end{abstract}

Palavras-Chave: Simulação por Computador, Educação Médica, Aprendizagem Ativa.

\begin{abstract}
This paper describes the development of simulation of clinical cases in a computerized environment on the web. The simulator was inserted into the Moodle and 18 students in the seventh semester of medicine performed the task and answered a validated questionnaire for the evaluation of software with questions about education: learning, interactivity, motivation, concentration, attractiveness, functionality and autonomy. The experience was considered extremely valuable. The challenge is enormous in order to seek alternatives that encourage interactivity and simultaneously harbor content, with a depth appropriate to the purpose of the course. Criticism in assessment will form the basis for adjustments in the next cases.
\end{abstract}

Keywords: Computer Simulation Medical Education, Problem-Based Learning. 


\section{Introdução}

Desde o século passado o ensino médico é fundamentado, na maioria das universidades, em um modelo tradicional, baseado em um paradigma biológico e mecanicista, onde se destaca o culto às doenças e não à saúde e onde se usa a tecnologia como simples forma de interpretação dos fenômenos vitais. A base do ensino, neste método, é geralmente segmentada em disciplinas não articuladas, sem a integração das ciências básicas e clínicas $[1,2]$.

Nos últimos anos tem-se visto diversas iniciativas dos Ministérios da Educação e da Saúde no sentido de mudar a educação médica e a saúde no Brasil. Como resultado, surgiram: as Diretrizes Curriculares Nacionais dos Cursos de Medicina, Enfermagem e Nutrição - Resolução CNE/CES, n. 4 [3], o Programa de Incentivo às Mudanças Curriculares das Escolas Médicas - PROMED [4], Programa Nacional de Reorientação de Formação Profissional em Saúde - PRÓ-SAÚDE [5], Programa de Educação para o Trabalho em Saúde - PET-SAÚDE [6] e Universidade Aberta do SUS - UNASUS [7].

$\mathrm{O}$ que pode ser percebido nas iniciativas do governo citadas no parágrafo anterior é o uso de uma estrutura curricular que perpasse as disciplinas, que seja orientada para a competência e possibilite um método ativo de ensino-aprendizagem centrado no estudante. Porém, em nenhuma destas iniciativas se percebe uma ênfase no uso de tecnologias para apoiar o ensino e aprendizagem.

Outro aspecto a ser destacado é o fato dos cursos de medicina ainda ensinarem a tratar a doença e não o doente. Esse modelo é chamado de biomédico ou reducionista [8], redução do ser humano a apenas um problema físico $[9,10]$. Tsuji e Silva [1] chamam a atenção para uma mudança prática da medicina, para que esta seja fundamentada no tratamento das pessoas e de seu entorno, na sua integralidade. Eles apresentam como representação do ser total o encontro das dimensões biológica (corpo), psicológica (mente) e social (meio ambiente).

$\mathrm{O}$ uso de métodos ativos pode ser uma boa alternativa, porém exigem um trabalho coletivo, onde o professor não é mais responsável somente por um conteúdo, desenvolvido para ser ministrado em uma disciplina independente, mas desenvolvida para o grupo, com a discussão permanente entre os pares. Rompe-se com o paradigma da educação fragmentada, onde o professor é o centro do conhecimento, e coloca-se a aprendizagem como núcleo do processo.

De acordo com as Diretrizes Curriculares Nacionais do curso de medicina, o currículo e o método pedagógico desejáveis devem propiciar o desenvolvimento da capacidade de observar, escutar, pensar e, consequentemente, aprender a aprender, ser, fazer e conviver com a autoaprendizagem. Os estudantes devem iniciar suas práticas desde o início do curso realizando atividades e tarefas de complexidade crescente.

Um dos grandes desafios do uso de métodos ativos de aprendizagem é a maneira de apresentar os problemas aos alunos, de forma que estes possam trabalhar e estudar os conteúdos ministrados a qualquer hora e em qualquer local. A simulação de casos reais parece adaptar-se bem como estratégia de desenvolvimento de conteúdos, onde o grupo de professores pode desenvolver casos clínicos, contemplando as diferentes disciplinas do currículo, e apresentando aos alunos em um ambiente digital.

A grande dificuldade é a criação destes casos, por diferentes razões: Complexidade no uso de simuladores; Desconhecimento de ferramentas tecnológicas para a criação das simulações; Grande número de simulações a serem criadas para contemplar todos os conteúdos; Interfaces diferentes para cada caso de estudo, de forma que motive o aluno a utilizar a ferramenta; Desenvolvimento de ambientes amigáveis aos alunos; Acompanhamento do aprendizado-evolução dos alunos com o uso das simulações.

A UFCSPA já tem implantado este sistema virtual e vem estimulando professores e alunos na utilização desta ferramenta no dia a dia escolar. O trabalho aqui apresentado propõe o uso de simulação desenvolvida com a ferramenta CourseLab. Este tem como vantagem o uso de um formalismo que permite a criação de simulações, onde o médico-professor não necessita conhecer informática para o desenvolvimento do material. O foco está na criação dos casos clínicos e não no conhecimento de programação ou tarefas complexas de informática.

Na seção dois é apresentada a simulação desenvolvida por um grupo de professores e alunos sobre um caso de Dor Lombar. A seção três contempla a análise sobre a experiência realizada com 18 alunos de uma turma de graduação em medicina, na disciplina de Nefrologia, seguida das considerações finais.

\section{Simuladores}

A partir da ideia de que a aprendizagem representa uma mudança de comportamento [11], buscam-se formas de prover experiência aos alunos. Nesta busca pelo conhecimento os simuladores têm representado um grande aliado, evoluindo e atraindo estudantes das mais variadas áreas do conhecimento. Busca-se, normalmente, nestas simulações, prover informações com características da vida real, permitindo a participação em cenários e situações próximos às vivenciadas no dia a dia.

Atualmente, não existem definições precisas sobre o 
termo simulação. Para Machado, Moraes e Nunes [12], estas visam apresentar situações práticas vivenciadas no dia-a-dia e tem como objetivo principal proporcionar o treinamento de profissionais, em várias áreas do conhecimento, situações críticas, conscientização de crianças, jovens e adultos.

Ziv et al. [13] definem simulação como uma "técnica em que se utiliza um simulador, considerando-se simulador como um objeto ou representação parcial ou total de uma tarefa a ser replicada".

De acordo com Bass [14] simulação se refere à aplicação de modelos computacionais para estudo e previsão de eventos ou comportamentos, disponibilizado para uma ampla gama de aplicações e sendo utilizada principalmente na área da educação.

No mesmo informe citado anteriormente é evidenciada a simulação na medicina como uma prática histórica.

Historicamente, o paradigma da medicina combina diagnóstico e empirismo, isto é, os médicos usam vários testes para diagnosticar uma condição médica e, em seguida, um plano de tratamento ou intervenção com base em dados empíricos e experiência profissional. Geralmente, a prática médica impede qualquer processo formal para prever o resultado de um tratamento para um paciente individual, embora possa haver alguns dados estatísticos para indicar a taxa de sucesso do tratamento.[14]

O uso de simulação permite novas abordagens para o ensino e prática médicas, denominadas de Medicina Baseada em Simulação. Por exemplo, os estudantes podem usar simulações anatômicas e fisiológicas para prever os resultados de procedimentos e, assim, acompanhar os resultados de tratamentos a pacientes virtuais.

Para fins de treinamento, os simuladores podem ser aplicados para apresentar situações críticas, que envolvam algum tipo de risco, tomada de decisões ou desenvolver habilidades específicas. Com base nisso, os simuladores para a área médica tem evoluído muito. Um erro no atendimento clínico ou cirúrgico pode ter como custo a vida de um paciente e, por isso, antes de atendê-los, um estudante de medicina deve ser exposto à maior quantidade de casos reais ou próximos à realidade na sua trajetória de preparação.

Em estudos de Muller [15] e Vozeniek et al [16] é evidenciado que a simulação pode ser aplicada tanto no ensino como na avaliação, pois em função de atividades práticas é possível avaliar tanto o conhecimento quanto a competência.

Bergeron [17] cita algumas características importantes no desenvolvimento de simuladores para a área da medicina, que são:
Precisão de conteúdo (Content accuracy): Estudo preciso sobre o tema abordado para que o usuário possa aprender conforme a realidade do caso estudado, ou seja, simulando realmente a vida real.

Conhecimento do domínio (Domain experts): Relacionado à precisão e a detalhes que devem ser observados no desenvolvimento do simulador, como, por exemplo, o som em uma ausculta cardíaca ou a exata coloração da pele em uma patologia.

Princípio do projeto (Design principles): O projeto deve refletir uma especificação de requisitos que define a interação dos usuários levando em consideração estilos, facilidade de uso, entre outros.

Pessoal (Staffing): A necessidade de especialização de domínio e precisão do conteúdo coloca exigências específicas sobre as necessidades de pessoal.

Padrões (Standards): A experiência da comunidade de ensino médico demonstra a necessidade de normas e padrões a serem seguidos de forma que esse conteúdo possa ser utilizado, compartilhado e disseminado.

Segundo Rankin e Vargas [18], muitas vezes os simuladores utilizam recursos gráficos avançados para envolver o usuário em um ambiente similar ao real para tomadas de decisão. Podem ser simulados: um ambiente, uma situação, uma ação e reação envolvendo um determinado assunto. Parte do pressuposto de que textos longos explicativos podem ser substituídos pela ilustração de processos. Normalmente, o simulador busca reproduzir fielmente a realidade, contextualizando o assunto e deixando o usuário explorar possibilidades em um ambiente semelhante ao encontrado na realidade.

$\mathrm{Na}$ área da saúde há exemplos de diversos tipos de simuladores. Na Tabela 1, são apresentados alguns exemplos retirados da literatura.

$\mathrm{O}$ aperfeiçoamento de simuladores para a área da saúde se dá, em grande parte, no uso e refinamento da Inteligência Artificial (IA). Nos simuladores a IA pode ser sumarizada como algoritmos que podem manipular conceitos, uso de heurística, representação do conhecimento, suporte a dados imprecisos, múltiplas soluções e integrar mecanismos de aprendizado de máquina [30].

De acordo com Bourg e Seemann [32] é possível dividir as técnicas de IA em dois grupos: determinísticas e não determinísticas. As primeiras são previsíveis, fáceis e rápidas de implementar, porém, a previsibilidade restringe a simulação, depois de algumas iterações o usuário perceberá quais os próximos estados e eventos. As não determinísticas facilitam a aprendizagem proporcionando uma simulação com final imprevisível. A dificuldade está na implementação e na depuração dos erros e testes de eventos específicos comparados aos determinísticos. Os 
tipos de simuladores com o uso de IA são divididos por Machado et al. [12] de acordo com sua atuação em dois níveis: controle de nível superior e o controle de nível inferior, este se referindo às decisões relacionadas ao enredo e aquele a decisões descentralizadas nas tomadas de decisão internas do simulador.

Estes sistemas, também conhecidos como baseados em conhecimento, possuem regras que reproduzem o conhecimento de especialistas, e são utilizados para solucionar problemas em domínios específicos. As principais características dos simuladores que utilizam IA são elencadas por Plemenos e Miaoulis [31] como:

- Manipulação de conceitos: são capazes de processar conceitos muito além de dados numéricos.

- Uso de heurísticas: utilizam métodos heurísticos para resolver problemas onde outra solução não pode ser obtida com o uso de algoritmos conhecidos. Os mé- todos heurísticos oferecem uma probabilidade elevada de encontrar uma solução considerada boa para determinados problemas.

- Representação do conhecimento: o conhecimento em programas com IA e representado de forma explícita.

- Permitir dados imprecisos: dependendo do método de tomada de decisão usado pode suportar dados imprecisos ou incompletos.

- Permitir múltiplas soluções: no caso de um dado e impreciso, a resolução do problema pode ser oferecida de diversas maneiras.

- Capacidade de aprender: sistemas inteligentes devem integrar mecanismos de aprendizado da máquina, buscando obter o raciocínio mais próximo possível da capacidade humana.

\begin{tabular}{|c|c|c|c|}
\hline Ênfase & Software/ Subject & Linguagem/Ambiente & Validação \\
\hline Casuais & $\begin{array}{l}\text { Happy Farm [19] } \\
\text { Controle glicêmico intensivo [20,21] }\end{array}$ & $\begin{array}{l}\text { Two-dimensional, side- } \\
\text { scrolling, "platform" games } \\
\text { and 3D environment }\end{array}$ & \\
\hline Habilidade & $\begin{array}{l}\text { Videolaparoscopic simulator [22] } \\
\text { The Emory Neuro Anatomy Carotid } \\
\text { Training (ENACT) }\end{array}$ & Realidade virtual & $\begin{array}{l}\text { Melhor desempenho de } \\
\text { tempo e redução de erros } \\
\text { Quantificação da curva de } \\
\text { aprendizado }\end{array}$ \\
\hline Estratégicos & $\begin{array}{l}\text { Cognitive forcing strategies in } \\
\text { Emergency Room [23] } \\
\text { Treinamento em Triagem de Inci- } \\
\text { dentes [24] }\end{array}$ & & $\begin{array}{l}\text { Assessment } \\
\text { exercise based on tagging } \\
\text { accuracy }\end{array}$ \\
\hline Simuladores & JDoc [25] & $\mathrm{C}++$ & $\begin{array}{l}\text { Usability } \\
\text { Questionnaire }\end{array}$ \\
\hline Treinamento & $\begin{array}{l}\text { Cardiopulmonary resuscitation }[26 \text {, } \\
27]\end{array}$ & $\begin{array}{l}\text { Massively multiplayer } \\
\text { virtual world }\end{array}$ & $\begin{array}{l}\text { A test-retest (6-month } \\
\text { interval between sessions) }\end{array}$ \\
\hline Educacionais & $\begin{array}{l}\text { Online virtual patients [28] } \\
\text { The Clinical Health Economics } \\
\text { System Simulation (CHESS) [29] }\end{array}$ & $\begin{array}{l}\text { Computerized team-based } \\
\text { quasi-competitive simulator }\end{array}$ & \\
\hline
\end{tabular}

Tabela 1: Exemplos de simuladores para a área da saúde.

\section{Simulação "Dor Lombar"}

A disciplina de Nefrologia é vinculada ao Departamento de Clínica Médica da Faculdade de Medicina da UFCSPA (Universidade Federal de Ciências da Saúde de Porto Alegre) e iniciou um projeto de criação de casos clínicos interativos a partir de casos reais atendidos na enfermaria de Nefrologia do hospital-escola (Santa Casa de Porto Alegre). O objetivo é que possam ser usados pelos alunos na forma de Ensino à Distância (EaD), ou seja, trabalhados em qualquer local e hora. Os casos clínicos são organizados de modo a constar da história, exame físico, dados de laboratório, imagem e anatomopatológicos em ambiente digital e com interatividade. A disposição do caso e de perguntas que vão sendo propostas ao longo do acesso ao caso, com links para textos curtos e informações adicionais permitem o estudo das principais patologias renais vivenciadas com casos reais, estimulando o raciocínio diagnóstico.

Com a simulação dos casos espera-se aproximar os alunos dos casos reais encontrados na sua atuação profissional, de modo atrativo e familiar, com o uso da tecnologia, estimulando o raciocínio clínico e instigando o aprofundamento do conhecimento sobre o tema proposto.

Esse projeto se inseriu no Programa de Iniciação à Docência com dois alunos bolsistas como integrantes da equipe, trazendo para a atividade o olhar dos próprios 
acadêmicos. A partir desse experimento em conjunto com o Departamento de Educação e Informação em Saúde pretende-se desenvolver o uso de ferramentas digitais que possam ser gradativamente disseminadas a outras disciplinas e cursos.

Duas ferramentas já foram desenvolvidas na categoria de Paciente Virtual. A categoria de Paciente Virtual, segundo Orton e Mulhausen [33], é assim definida: " $P a$ ciente Virtual é um programa interativo que simula a vida real em cenários clínicos, que permite o aprendizado de atos do profissional da saúde, obtendo a história clínica, exames e realizando diagnóstico e decisões terapêuticas".

A primeira ferramenta é denominada SIACC (Sistema Interdisciplinar de Análise de Casos Clínicos). Este projeto foi dividido em quatro fases: especificação de requisitos, definição da arquitetura, codificação e implantação. Para a realização dessas fases foi necessário contar com a colaboração de docentes dos departamentos de Patologia, Cirurgia e Clínica Médica da Faculdade de Medicina da UFCSPA, envolvendo os serviços de Nefrologia, Coloproctologia e Gineco-obstetrícia do hospital de ensino.

Os casos modelados no SIACC devem ser organizados de modo a constar de: história, exame físico, dados de laboratório e imagens. A disposição do conteúdo e de perguntas propostas no decorrer do caso, com links para textos curtos e informações adicionais, deve permitir o estudo das principais patologias vivenciadas na atuação profissional médica, estimulando o raciocínio diagnóstico. Mais informações sobre o SIACC podem ser encontradas em [34].

A segunda ferramenta é denominada SimDeCS (Simulador Inteligente para a Tomada de Decisão em Cuidados de Saúde). O ambiente SimDeCS deve permitir aos alunos de medicina a análise de vários casos clínicos, apoiando sua aprendizagem, facilitando o desenvolvimento de suas habilidades técnicas e competências sobre diagnósticos formulados

Diferente do SIACC, o SimDeCS apresenta os casos clínicos no formato de um jogo sério, onde os alunos podem acompanhar o estado de saúde do paciente, realizar a anamnese, obter os exames físico e complementares e, a partir de evidências apresentarem um diagnóstico, seguido de uma ou mais condutas.

O SimDeCS se utiliza de formalismos de Inteligência Artificial que conferem estrutura robusta, com diretrizes clínicas armazenadas em redes bayesianas, acompanhamento do aluno por meio de um sistema multiagente e estratégias pedagógicas disparadas ao longo da execução do caso, com base na decisão de um diagrama de influência sobre a melhor estratégia em cada passo da resolução do caso clínico. A decisão da melhor estratégia é baseada na confiança declarada pelo aluno e pelo acompanhamen- to de um agente sobre as atitudes do mesmo no simulador. Mais informações sobre o SimDeCS podem ser encontradas em [35][36][37][38].

$\mathrm{Na}$ sequência é apresentado o primeiro experimento, que deu origem às ferramentas descritas anteriormente. Trata-se de um relato de caso que usou método descritivo e tabelas de frequência para descrição dos achados.

O primeiro experimento foi desenvolvido usando como modelo o caso interativo "Bitter Pill" disponibilizado pelo periódico médico The New England Journal of Medicine. Este pode ser acessado em: http://www. nejm.org/doi/full/10.1056/NEJMimc1004455.

A ferramenta utilizada para o desenvolvimento desta simulação foi o software livre denominado CourseLab associado ao uso de outros softwares para melhoria de imagens e vídeos que tiveram que ser importadas. Os vídeos utilizados foram obtidos já prontos da Web.

A simulação foi estruturada de forma que contivesse todos os elementos de uma consulta clínica e o seu seguimento: identificação do paciente (ID), queixa principal (QP), coleta da história médica (HM), exame físico (EF), solicitação de exames subsidiários (ES), conduta (CD) e acompanhamento. Foram inseridos testes em diversas etapas (a que são designados escores parciais, que são somados ao final da simulação para compor o escore final) e informações adicionais, que foram fornecidas compulsoriamente ou puderam ser consultadas opcionalmente.

\subsection{Desenvolvimento da Simulação}

Na sequência são apresentadas imagens da simulação desenvolvida no Courselab, juntamente com explicações de como foi estruturada cada interface desta.

O CourseLab é uma ferramenta de autoria gratuita que permite a geração e exportação de conteúdos no formato padrão SCORM. O editor organiza o conteúdo produzido em um formato similar a produção de slides (estilo Power Point). Com essa ferramenta é possível criar um conjunto de módulos que podem ser importados e utilizados em Ambientes Virtuais de Aprendizagem AVA (Moodle e Blackboard, por exemplo).

O sistema dispõe de muitos recursos prontos, além do suporte a criação de animações. Possui múltiplas formas de conteúdo, bem como a possibilidade de ser utilizado para a criação de atividades e questionários. $\mathrm{O}$ resultado do desempenho dos alunos pode ser enviado automaticamente para um AVA, em função do uso do padrão SCORM.

Um diferencial do CourseLab para outras ferramentas de autoria é a possibilidade do uso de agentes nos módulos ou materiais pedagógicos desenvolvidos. Existem 
configurações que podem ser aplicadas a esses agentes, permitindo que eles tomem ações específicas com base nas interações do aluno com a ferramenta.

Inicialmente foi desenvolvida a tela de apresentação, uma capa indicando a autoria do trabalho. Ao clicar no botão iniciar, aparecerá uma tela contendo a identificação do paciente, a queixa principal e uma descrição da doença (figura 1).

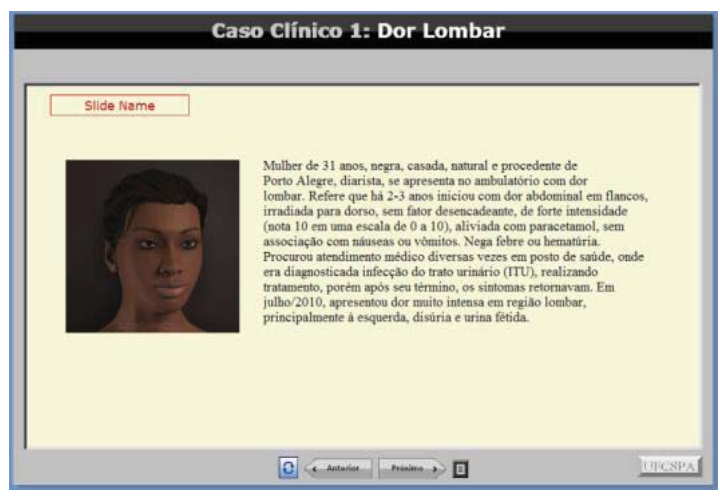

Figura 1: Identificação, queixa principal e descrição da doença do paciente

Após a descrição de outros aspectos da história médica do paciente, é apresentada uma tela contendo uma representação do corpo do paciente, com pontos vermelhos indicando aspectos importantes do exame físico que podem ser observados ao se passar o mouse sobre estes. (figura 2).

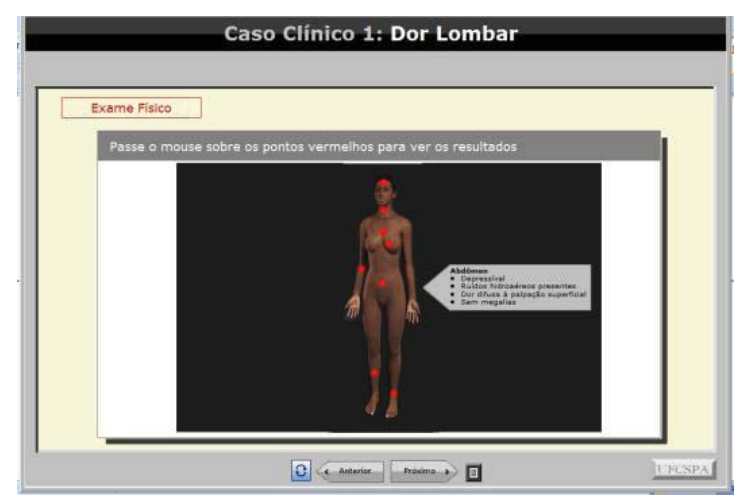

Figura 2: Representação de resultados de exames físicos

Cabe ao professor, na construção do caso clínico, informar aspectos importantes a serem apresentados aos alunos referentes a cada aspecto analisado no exame físico.

A figura 3 apresenta um teste de múltipla escolha sobre as possíveis hipóteses diagnósticas. $\mathrm{O}$ aluno deve selecionar uma ou mais hipóteses e clicar no botão para envio das respostas. É apresentada a correção da questão enviada, bem como respostas, comentários e sugestões de leituras complementares. Neste momento, um escore parcial é atribuído ao aluno.



Figura 3: Exemplo de teste

O feedback que o aluno receberá em cada questão deve ser pensado com parcimônia, pois pode levá-lo a compreender melhor aspectos importantes do caso. Muito mais do que indicar se uma questão está correta ou não, é importante conduzir o aluno a entender o porquê do seu erro ou acerto.

São disponibilizados outros testes, como referentes a exames complementares a serem solicitados e em cada um, novo escore parcial é armazenado. Os resultados dos exames que deveriam ter sido solicitados são apresentados, contendo texto, imagem e som, dependendo do tipo de exame. A figura 4 apresenta um exemplo de exame solicitado e seu diagnóstico.

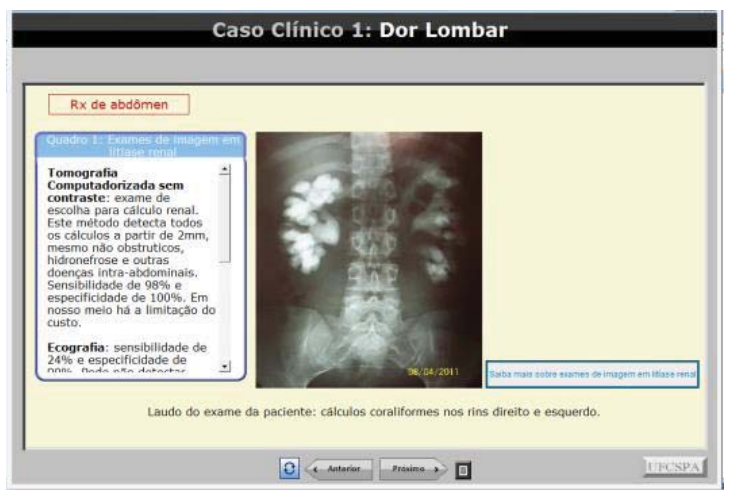

Figura 4: Resultado de exame solicitado

O exame pode ser apresentado na forma de imagem ou som, porém, é sempre importante que seja acompanhado de um texto explicativo, para que o aluno possa aprender a analisá-lo e entender suas características.

O acompanhamento do caso do paciente é mostrado em telas subsequentes e novos testes de conhecimentos aplicados aos alunos. Um exemplo disso é apresentado na figura 5, onde cada opção para tratamento fornecido para o aluno é apresentado com um link que possibilita a visita de locais contendo vídeos sobre o procedimento do tra- 
tamento, como neste caso, de nefrolitotomia percutânea.

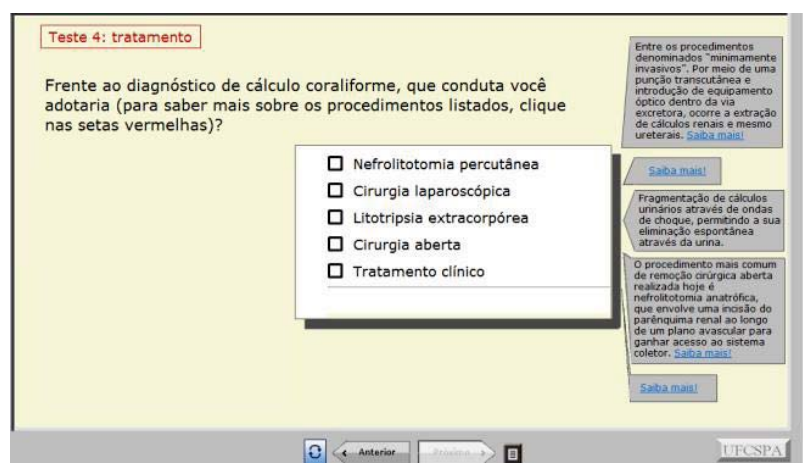

Figura 5: Teste sobre conduta a ser seguida

Ao final da simulação é apresentado o tratamento que deveria ser indicado ao paciente, com explicações pertinentes ao caso. Ao encerrar a simulação, um escore final é apresentado ao aluno.

\subsection{Experimento realizado com o simulador}

O simulador foi aplicado a uma turma de alunos da $4^{\mathrm{a}}$ série do curso de Medicina da UFCSPA, durante a disciplina de Nefrologia, num período de 1h, no Laboratório de Informática. O simulador foi inserido na Plataforma Moodle e acessado pelos alunos em aula. No final da atividade, os mesmos responderam a um questionário, com 15 perguntas, também disponibilizado no ambiente Moodle. Este questionário foi elaborado seguindo orientações das seguintes referências bibliográficas: Brasil [39] e Monteiro [40].

O questionário abordou questões relativas ao desenvolvimento dos alunos, interatividade, motivação, tempo de execução, usabilidade e aprofundamento científico.

\section{Análises da aplicação da simulação}

A turma onde foi aplicado o trabalho era composta por 18 alunos. Um dos respondentes encaminhou o questionário em branco, não tendo suas respostas computadas na análise.

A primeira questão buscava a percepção do aluno quanto à elaboração do exercício, se esse fora projetado visando o desenvolvimento do aluno. Dos 17 respondentes, um aluno $(5,88 \%)$ discordou totalmente, três alunos concordaram parcialmente $(17,65 \%)$ e a grande maioria, 13 alunos, concordou totalmente com essa afirmação (76,47\%). Um dos alunos, que discordou parcialmente, inseriu um comentário na questão sugerindo que para haver um novo aprendizado deveria haver mais informações sobre as questões certas e erradas. Destacou ainda que o foco estava mais em testar conhecimentos do que desenvolver o mesmo.
A segunda questão tinha como ênfase a percepção do estímulo à interatividade do aluno com o uso de ferramentas tecnológicas para a resolução dos problemas propostos em aula. Quatro alunos $(23,53 \%)$ consideraram regular, oito alunos $(47,06 \%)$ consideraram bom e cinco alunos $(29,41 \%)$ consideraram muito bom. Nenhum aluno considerou muito ruim ou ruim. O gráfico a seguir ilustra o achado.

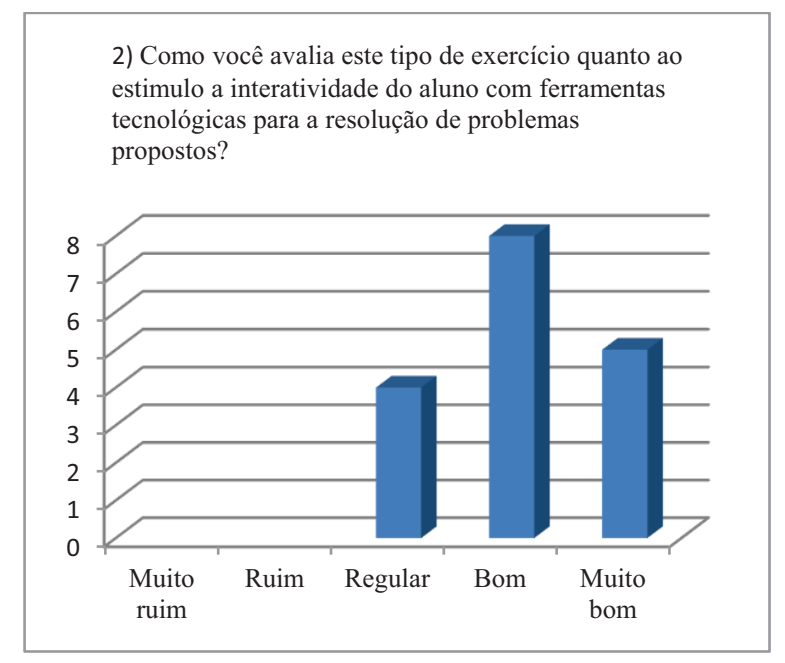

Figura 6: Estímulo à interatividade com ferramentas tecnológicas

Esta questão chama a atenção quanto à percepção do aluno em relação ao uso de tecnologias. Por ser um público alvo composto por jovens, acostumados com o uso das tecnologias no seu dia a dia, esperava-se um percentual bastante alto na opção muito bom. A questão que se pode fazer a partir da realidade observada nas respostas é que: na área educacional não estão acostumados a usar estes recursos? A ferramenta não foi suficientemente atrativa?

Quando questionados sobre a capacidade do exercício em motivá-los e concentrá-los na resolução do problema proposto, a realidade foi bastante semelhante à questão anterior. Dos 17 respondentes, sete $(41,18 \%)$ avaliaram como regular, oito $(47,06 \%)$ consideraram boa e dois $(11,76 \%)$ muito boa. Um dos respondentes ainda destacou em sua resposta que a conversa em aula prejudica a concentração e por isso prefere trabalhar em casa.

A questão quatro tinha como objetivo descobrir se o aluno, no decorrer do exercício, havia se sentido pressionado ou obrigado a avançar nas tarefas mesmo sem ter compreendido na íntegra o problema apresentado. Nenhum respondeu ter se sentido obrigado ou pressionado, três alunos $(17,65 \%)$ responderam que parcialmente e 14 alunos $(82,35 \%)$ responderam que não. Cabe destacar que um dos alunos que respondeu parcialmente, indicou sentir-se pressionado pela ansiedade de descobrir as respostas. O gráfico a seguir apresenta as respostas recebidas 
dos alunos.

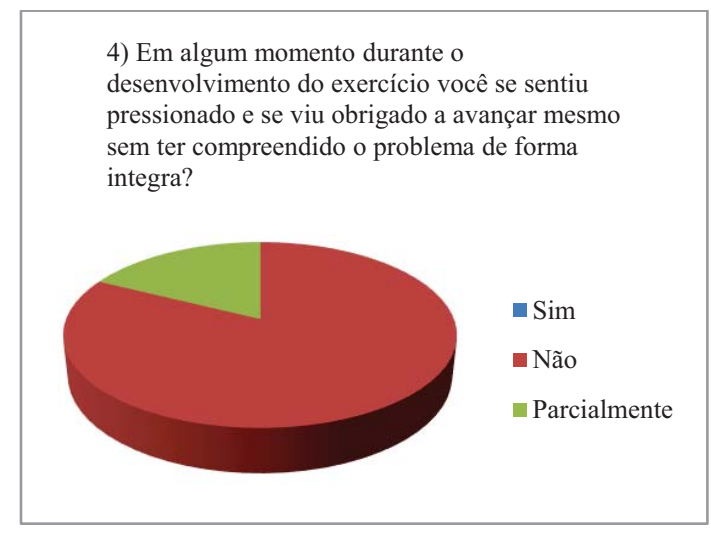

Figura 7: Obrigação ou pressão para seguir sem ter compreendido o problema

Quanto à capacidade do exercício em gerar autonomia na aprendizagem permitindo aos alunos conduzir seus estudos sozinhos, no ritmo que lhe convier, todos os alunos concordaram, com 13 alunos $(76,47 \%)$ dizendo que o exercício é bom neste sentido e quatro $(23,53 \%)$ que é muito bom. Nenhum aluno respondeu muito ruim, ruim ou regular.

Sobre a relevância e a contextualização do conteúdo abordado para o aprendizado todos os alunos consideraram relevante ou muito relevante para a sua aprendizagem. Chamou à atenção a resposta descritiva fornecida por um dos respondentes que diz: "Penso que é uma forma de abordagem mais abrangente resolvendo um caso clínico, como se tivéssemos acompanhando o paciente desde o início de sua enfermidade".

Todos os alunos concordaram em suas respostas que o exercício realizado proporciona um aprofundamento científico sobre o assunto abordado, destacando-se a resposta de um enfatizando que "a maior relevância do exercício é o teste de conduta clínica frente a um caso".

A grande maioria, representada por 15 alunos $(88,24 \%)$, indicou que o exercício realizado apresentou em seu conteúdo bibliografias relevantes para a continuidade do aprendizado e da pesquisa sobre o tema abordado.

Foram apresentadas quatro questões que avaliavam os aspectos visuais e interativos do trabalho proposto. $\mathrm{O}$ gráfico mostrado na figura 8 apresenta as questões e respectivas respostas.

É possível perceber no gráfico da figura 8 que estes aspectos foram muito bem avaliados pelos alunos. $\mathrm{O}$ itens visual, facilidade de uso, atratividade de uso foram considerados, em sua maioria, bons ou muito bons. Chama a atenção que o aspecto navegabilidade mereceu destaque como bom, o que significa que esse aspecto deve ser reavaliado, buscando ainda melhorias.

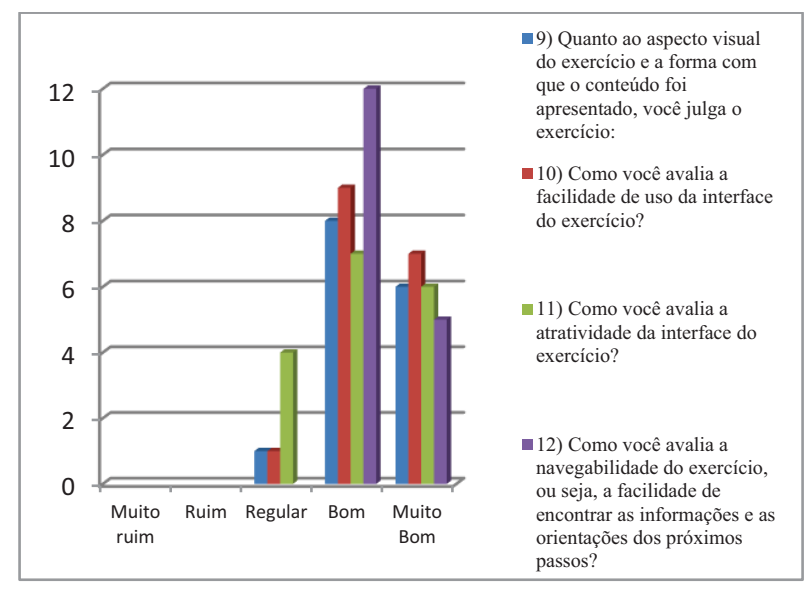

Figura 8: Aspectos visuais e interativos

Quanto ao tamanho do exercício, apenas um aluno respondeu que achou longo. Em relação ao local para resolver o exercício, a maioria, 13 alunos $(76,47 \%)$ prefere respondê-lo em casa, alegando maior liberdade e tempo para consultas bibliográficas.

Algumas críticas foram inseridas nas respostas dos alunos, destacando-se a questão do tempo para responder durante a aula, não permitindo ao aluno retomar algumas questões, bem como a falta de explicação quanto à possibilidade de haver mais de uma alternativa correta ou o fato de considerar as questões incompletas como incorretas.

$\mathrm{Na}$ parte operacional, destacaram-se respostas como: erros no Moodle ao abrir imagens do exercício e, principalmente, o fato do exercício só funcionar corretamente no Internet Explorer, quando muitos alunos utilizam outros navegadores, como o Firefox ou Chrome.

\section{Considerações finais}

Um dos desafios no uso de métodos ativos de aprendizagem é apresentar os problemas aos alunos, de forma que estes possam trabalhar e estudar os conteúdos a qualquer hora e local. No curso de medicina a simulação de casos reais parece adaptar-se bem como estratégia de desenvolvimento de conteúdos.

O trabalho aqui apresentado descreve o desenvolvimento de simulação de um caso clínico em um ambiente digital na web. O princípio norteador do presente trabalho é aproximar os estudantes de casos reais numa apresentação próxima da realidade virtual a que estão habituados, de modo atrativo e que permita estimular o raciocínio clínico e instigar o aprofundamento do conhecimento sobre o tema proposto.

A proposta é a criação de casos interativos a partir de 
casos reais atendidos na enfermaria de Nefrologia. A disposição do caso e de perguntas que vão sendo propostas ao longo do acesso ao caso, com links para textos curtos, informações adicionais e vídeos, permitiram o estudo de uma patologia renal comum, a partir de um caso real em um ambiente digital amigável de modo a facilitar o estudo e o raciocínio diagnóstico.

Os recursos gráficos da simulação e interatividade, com inclusão de testes e textos informativos foram criados utilizando recursos do software Courselab além da importação de imagens e vídeos. A simulação foi estruturada na forma de uma consulta clínica: identificação, queixa principal, história da doença, exame físico, exames complementares, diagnóstico, conduta e desfecho. Foram inseridos em cada etapa, testes (a que foram designados escores parciais, somados ao final para compor o escore final) e informações adicionais, que foram fornecidas compulsoriamente ou puderam ser consultadas opcionalmente para aprofundamento do assunto.

O simulador foi inserido no Moodle e 18 alunos do 7o semestre de medicina realizaram a tarefa e responderam a um questionário validado para a avaliação de softwares educativos com perguntas sobre: o aprendizado, a interatividade, motivação, concentração, atratividade, operacionalidade e autonomia.

Consideramos a experiência extremamente válida. A participação dos alunos no planejamento e execução imprimiu a perspectiva desejada para atingir o alvo a quem a atividade se destina. O desafio é enorme no sentido de buscar alternativas de interatividade que estimulem e que simultaneamente abriguem conteúdo, com um aprofundamento adequado ao objetivo do curso e ainda a apropriação das diferentes ferramentas tecnológicas para serem utilizadas na construção de casos. As críticas na avaliação servirão de base para ajustes nos próximos casos.

Além disso, o projeto serviu de base para o desenvolvimento de dois simuladores do tipo paciente virtual (SIACC e SimDeCS) que já estão em uso na UFCSPA e sendo validados como ferramentas que permitam a interatividade dos alunos e o aprimoramento do raciocínio diagnóstico dos mesmos.

\section{Referências}

[1] H. Tsuji, R. H. Silva. Aprender e ensinar na escola vestida de branco: do modelo biomédico ao humanístico. São Paulo: Phorte, 240p, 2010.

[2] J. B. Lampert. Tendências de Mudanças na formação médica no Brasil - Tipologia das escolas. São Paulo: Hucitec/ABEM. 2002.
[3] Brasil. Conselho Nacional de Educação. Câmara de Educação Superior. Resolução CNE/CES 4/2001. Diário Oficial da União, Brasília, 9 de novembro de 2001. Seção 1, p. 38. Disponível em http://portal.mec.gov.br/cne/arquivos/pdf/ CES04.pdf. Acesso em 02/11/2010.

[4] Brasil. Programa de Incentivo às Mudanças Curriculares das Escolas Médicas - PROMED. Brasília - DF: Ministério da Saúde e Ministério da Educação. 2001. Disponível em http://www.famema.br/documentos/programa_pr omed.pdf. Acesso em 07/11/2010.

[5] Brasil. Programa Nacional de Reorientação Profissional e Saúde - PRÓ-SAÚDE. Brasília DF: Ministério da Saúde e Ministério da Educação. $2005 . \quad$ Disponível em: http://www.famema.br/documentos/prosaude.pdf Acesso em 07/11/2010.

[6] Brasil. Programa de Educação pelo Trabalho para a Saúde - PET-SAÚDE. Brasília - DF: Portaria Interministerial MS/MEC n. 1.802, de 26 de agosto de 2008. Diário Oficial da União, 27/08/08; Seção 1:27. Disponível em: http://bvsms.saude.gov.br/bvs/saudelegis/gm/20 08/pri1802_26_08_2008.html. Acesso em 07/11/2010.

[7] Brasil. Universidade Aberta do SUS - UNASUS - Projeto Executivo. Brasília - DF: Ministério da Saúde. 2009. Disponível em: http://sites.google.com/a/universidadeabertadosu s.org.br/home/home. Acesso em 07/11/2010.

[8] J. E. DesMarchais, M. A. Bureau, B. Dumais, G. Pigeon. From traditional to problem-based learning: a case report of complete curriculum reform. Medical Education. 26: 190-9, 1992.

[9] R. M. Bentz. La educación médica de postgrado en la Argentina: el desafío de una nueva práctica educativa. Organización Panamericana de la Salud-OPAS. 336p, 2005.

[10] O. Pustal. A "ordem" de vida para o medico pensar o paciente: uma ontologia espinosana de ser humano na medicina. Tese de Doutorado, Universidade Federal do Rio Grande do Sul, Faculdade de Educação, Outubro 2006.

[11] A. S. Giusta. Concepções de Aprendizagem e Práticas Pedagógicas. Educação em Revista. 1: 24-31, 1985.

[12] L. Machado, R. Moraes, F. Nunes. Serious Games para Saúde e Treinamento Imersivo. In Fatima L. S. Nunes; L. S. Machado, M. S. Pinho, C. Kirner, (eds.) Abordagens Práticas de Reali- 
dade Virtual e Aumentada. Porto Alegre: SBC, páginas 31-60, 2009.

[13] A. Ziv, P. R. Wolpe, S. D. Small, S. Glick. Simulation-based medical education: an ethical imperative. Academic Medicine. 78(8):783-8, 2003.

[14] J. Bass. Revolutionizing Engineering Science through Simulation. A Report of the National Science Foundation Blue Ribbon Panel on Simulation-Based Engineering Science, 2006.

[15] G. E. Muller. The assessment of clinical skills / competence / performance. Academic Medicine. 65, suppl. 9:S63-S7, 1990.

[16] J. Vozenilek, J. S. Huff, M. Reznek, J. A. Gordon. See one, do one, teach one: advanced technology in medical education. Academic Emergency Medicine. 11(11):1149-54, 2004.

[17] B. P. Bergeron. Developing serious games. Massachusetts: Charles River Media. 2006.

[18] J. R. Rankin, S. S. Vargas. A Review of Serious Games and other Game Categories for Education. Melbourne, 2008.

[19] L. G. Gamberini, F. Marchetti, F. Martino, A. Spagnolli. Designing a serious game for young users: the case of happy farm. Studies in health technology and informatics. 144:77-81, 2009.

[20] D. Thompson, T. Baranowski, R. Buday, J. Baranowski, M. Juliano, M. Frazior, J. Wilsdon, R. Jago. In pursuit of change: youth response to intensive goal setting embedded in serious video game. Journal of Diabetes Science and Technology. 1(6):907-17, 2007.

[21] D. Thompson, T. Baranowski, R. Buday, J. Baranowski, M. Juliano, M. Frazior, J. Wilsdon, R. Jago, V. Thompson, M. J. Griffith. Serious Video Games for Health: How Behavioral Science Guided the Development of a Serious Video Game. Simulation \& Gaming. 41:587, 2010.

[22] N. E. Seymour, et al. Virtual reality training improves operating room performance. Annals of Surgery. 236:458-64, 2002.

[23] W. F. Bond, L. M. Deitrick, D. C. Arnold, M. Kostenbader, G. C. Barr, S. R. Kimmel, C. C. Worrilow. Using simulation to instruct emergency medicine residents in cognitive forcing strategies. Academic Medicine. 79(5): 438-46, 2004.

[24] J. F. Knight, S. Carley, B. Tregunna, S. Jarvis, R. Smithies, J. Huizinga. Homo Ludens: o jogo como elemento da cultura. São Paulo: Perspectiva, 2000 .
[25] A. Sliney, D. Murphy. JDoc: A Serious Game for Medical Learning. In First International Conference on Advances in Computer-Human Interaction. s.1.: IEEE Computer Society, 2008.

[26] J. Creutzfeldt1, L. Hedman, C. Medin, L. Heinrichs, L. Felländer-Tsai. Exploring Virtual Worlds for Scenario-Based Repeated Team Training of Cardiopulmonary Resuscitation in Medical. Journal of Medical Internet Research. 12(3), 2010.

[27] J. Creutzfeldt, L. Hedman, C. Medin, C. J. Wallin, A. Hendrick, P. Youngblood, W. L. Heinrichs, L. Felländer-Tsai. Implementing virtual worlds for systematic training of prehospital CPR in medical school. Studies in health technology and informatics. 125, 2007.

[28] D. Dewhust, E. Borgstein, M. E. Grant, M. Begg. Dewhurst D, Borgstein E, Grant ME, Begg M. Online virtual patients - A driver for change in medical and healthcare. Medical Teacher. 31(8):721-4, 2009.

[29] J. D. Voss, M. M. Nadkarni, J. M. Schectman, J. M. The clinical health economics system simulation (CHESS): A teaching tool for systems- and practice-based learning. Academic Medicine. 80(2):129-34, 2005.

[30] A. M. Morais, A. S. Souza, L. S. Machado, R. M. Moraes. Tomada de Decisão aplicada à Inteligência Artificial em Serious Games voltados para Saúde. In IX Encontro Regional de Matemática Aplicada e Computacional. João Pessoa, 2009.

[31] D. Plemenos, G. Miaoulis. Visual Complexity and Intelligent Computer Graphics Techniques Enhancements. Springer-Verlag New York Inc. Berlin Heidelberg. 2009.

[32] D. Bourg; G. Seemann. AlI for game developers. O'Reilly. 2004.

[33] E. Orton; P. Mulhausen, P. E-learning virtual patients for geratric education. Gerontology \& Geriatrics Education, 28(3): 73-88, 2008.

[34] R. L. Sebastiani; M. K. Zago; A. Montovani; M. R. Bez; R. M. Bruno; A. Dahmer; C. D. Flores. Ferramenta de Autoria para Construção de Casos Clínicos Interativos para Educação Médica.. In XII Workshop de Informática Médica. Porto Alegre: SBC - Sociedade Brasileira de Computação. 1:1-10, 2012.

[35] M. R. Bez; C. D. Flores; J. M. L. Fonseca; V. Maroni; P. R. Barros; R. M. Vicari. Influence Diagram for selection of pedagogical strategies 
in a multi-agent system learning. Advances in Artificial Intelligence-IBERAMIA. Lecture Notes in Computer Science. 7637:621-630, 2012.

[36] V. Maroni; C. D. Flores; S. C. Cazella; M. R. Bez; A. Dahmer. Development and Evaluation of an Intelligent Pedagogical Agent for the SimDeCS software. Procedia Technology 9:12171226, 2013.

[37] C. D. Flores; M. R. Bez; A. Respício; J. M. Fonseca. Training Clinical Decision-Making through Simulation. Decision Support Systems - Collaborative Models and Approaches in Real Environments. Lecture Notes in Business Information Processing. 121:59-73, 2012.

[38] P. R. M. Barros; S. C. Cazella; M. Bez; C. D. Flores; A. Dahmer; J. B. Mossmann; J. M. Fonseca; V. Maroni. Um Simulador de Casos Clínicos Complexos no Processo de Aprendizagem em Saúde. RENOTE - Revista Novas Tecnologias na Educação. 10(1):1-11, 2012.

[39] L. M. Brasil (org.) "Informática em Saúde". Brasília: Editora Universa. 574p, 2008.

[40] M. I. N. L. Monteiro. Avaliação de software educativos: aspectos relevantes. Revista ECurriculum. 2(2), 2007. 